\title{
ESTOQUE DE CARBONO DO SOLO SOB PASTAGEM EM ÁREA DE TABULEIRO COSTEIRO NO SUL DA BAHIA ${ }^{(1)}$
}

\author{
Oldair Vinhas Costa ${ }^{(2)}$, Reinaldo Bertola Cantarutti ${ }^{(3)}$, Luiz Eduardo \\ Ferreira Fontes $^{(4)}$, Liovando Marciano da Costa ${ }^{(4)}$, Paulo Gabriel \\ Soledade Nacif ${ }^{(2)} \&$ José Cláudio Faria ${ }^{(5)}$
}

\begin{abstract}
RESUMO
A substituição de ambientes de floresta por pastagens leva a importantes mudanças no estoque de $\mathbf{C}$ do solo e consequentemente na ciclagem global desse elemento. Este trabalho foi realizado no município de Itabela - Bahia, e teve por objetivo avaliar o $\mathrm{C}$ estocado no solo em pastagem degradada e produtiva com diferentes idades de uso, comparativamente ao solo de mata natural. Para tal, foram selecionadas áreas com remanescentes de Mata Atlântica, pastos produtivos com 2,9 e 18 anos de uso e pasto mal manejado com 18 anos, situadas em relevo plano e mesma classe de solo (Argissolo Amarelo). Nesses locais, foram alocadas três parcelas para coletas de amostras de solo e posterior análise de C. Com os dados obtidos, foi possível observar que não houve diferença significativa para os estoques de $\mathrm{C}$ do solo, entre os ambientes de mata, pasto degradado e pasto produtivo, com diferentes idades de uso e nas diferentes camadas de solo avaliadas. Além disso, observou-se que após 28 anos de uso com pastagem bem e mal manejada, em média 62 \% do C orgânico do solo ainda é derivado da floresta original.
\end{abstract}

Termos de indexação: Mata Atlântica, isótopos de C, manejo de pastagem.

\footnotetext{
(1) Parte da Tese de Doutorado apresentada pelo primeiro autor ao Curso de Pós-Graduação em Solos e Nutrição de Plantas, Universidade Federal de Viçosa - UFV. Apoio financeiro CNPq. Recebido para publicação em julho de 2008 e aprovado em junho de 2009.

(2) Professor Adjunto do Centro de Ciências Agrárias, Ambientais e Biológicas, Universidade Federal do Recôncavo da Bahia UFRB. CEP 44380-000 Cruz das Almas (BA). E-mails: oldair@ufrb.edu.br; pgabriel@ufrb.edu.br

(3) Professor Associado do Departamento de Solos, Universidade Federal de Viçosa - UFV. Av. Peter Henry Rolfs s/n, CEP $36570-$ 000 Viçosa (MG). E-mails: cantarutti@ufv.br

(4) Professor Titular Departamento de Solos, UFV. E-mails: luizfontes@ufv.br; liovandomc@yahoo.com.br

(5) Professor Adjunto da Universidade Estadual de Santa Cruz - UESC. Rod. Ilhéus/Itabuna, Km 16, CEP $45650-000$ Ilhéus (BA). E-mail: joseclaudio.faria@gmail.com
} 


\title{
SUMMARY: SOIL CARBON STOCKS UNDER PASTURE IN COSTAL TABLELAND AREAS IN SOUTHERN BAHIA STATE, BRAZIL
}

\begin{abstract}
The replacement of forest environments by pastures leads to fundamental alterations in soil carbon stocks and consequently in the global cycle of this element. This study was conducted in the municipality of Itabela, Bahia, and the objective was to evaluate carbon stocks in soils of a natural forest in comparison to degraded and productive pastures with different usage periods. For this purpose, remnants of the Atlantic Forest, productive pastures after different periods of use (2, 9 and 18 years) and an ill-managed pasture (18 years) were selected. All areas were located on a flat terrain of the same soil class (Typic Paleudult). In each area three plots were established and used for soil sampling and subsequent carbon analyses. Results showed no significant difference in soil carbon stocks in the forest and degraded or productive pastures with different use periods and soil layers. Moreover, it was observed that after 28 years of usage an average of the $62 \%$ of organic carbon found in well and ill-managed pastures was still derived from the original forest soil organic matter.
\end{abstract}

Index terms: Atlantic forest, carbon isotopes, pasture management.

\section{INTRODUÇÃO}

As perturbações causadas pelo homem ao ciclo de $\mathrm{C}$ têm sido diretas e indiretas. Um dos efeitos diretos claros é a modificação da estrutura e distribuição da vegetação por meio de alterações no uso da terra. A eliminação da vegetação florestal e sua substituição por outra cobertura superficial produzem efeitos negativos no ciclo de $\mathrm{C}$, mediante a perda de capacidade fotossintética na vegetação florestal e a liberação simultânea de grandes quantidades de $\mathrm{C}$ acumuladas nos ecossistemas florestais ao longo do tempo (Apps, 2003).

A preocupação com a mudança climática global e com as medidas mitigadoras do problema, principalmente envolvendo ciclagem e fixação de C, tem colocado o sistema pastoril como um dos grandes responsáveis por alterações negativas no meio ambiente. Isso se deve, principalmente, ao fato desse sistema aparecer, em geral, em substituição a ambientes naturais de mata, havendo, com isso, grandes liberações de $\mathrm{C}$, principalmente devido às práticas de formação dos pastos, em especial quando se faz uso de queimadas.

Apesar das críticas, ainda não há dados conclusivos a respeito da quantidade de $\mathrm{C}$ que é liberada com a substituição de mata nativa por sistemas de pastagens, nem mesmo quanto de C é mantido nos diversos compartimentos desse novo ambiente, principalmente se tratando do compartimento solo. Nesse caso, a substituição de ambientes naturais por agroecossistemas com culturas introduzidas causa alteração tanto na quantidade quanto na qualidade do $\mathrm{C}$ do solo.

Segundo Corazza et al. (1999), o conhecimento do estoque de $\mathrm{C}$ e de sua dinâmica no solo em sistemas naturais e agroecossistemas é importante no desenvolvimento de tecnologias para estabelecer sistemas sustentáveis, bem como para analisar o papel do solo como fonte ou depósito de $\mathrm{C}-\mathrm{CO}_{2}$ para a atmosfera.

A utilização de traçadores isotópicos naturais é um método bastante utilizado para distinguir e quantificar a origem da matéria orgânica nos solos cultivados. A abundância natural de ${ }^{13} \mathrm{C}$ na matéria orgânica do solo permite examinar a matéria orgânica derivada da floresta e da pastagem no balanço de carbono nos solos (Cadisch et al., 1996).

Nesse sentido, este trabalho foi proposto com o objetivo de avaliar o C estocado no solo, bem como a sua origem em solo de pasto degradado e pasto produtivo com diferentes idades de uso, em comparação com a mata natural.

\section{MATERIAL E MÉTODOS}

Este trabalho foi desenvolvido na Estação Experimental de Zootecnia do Extremo Sul (ESSULCEPEC/CEPLAC), localizada no município de Itabela $\left(16^{\circ} 39^{\prime} \mathrm{S}-39^{\circ} 30^{\prime} \mathrm{W}\right)$, região do extremo sul da Bahia. Este município está dentro do domínio da Mata Atlântica, em área de transição entre os climas Am (tropical de monção) e Af (tropical chuvoso de floresta), segundo a classificação de Köppen, com precipitação anual média de $1.311 \mathrm{~mm}$ e temperatura média de $25^{\circ} \mathrm{C}$, sem estação seca definida. Segundo CEPLAC (1983), na região predomina o relevo plano (tabuleiro) a suave ondulado, apresentando dissecamentos com vales em 'V", e os solos predominantes são os Argissolos Amarelos desenvolvidos a partir de sedimentos argiloarenosos do Terciário (Formação Barreiras).

Para a realização do trabalho, foram selecionadas, na estação, áreas em relevo plano em que o solo era um Argissolo Amarelo Distrófico abrúptico (Embrapa, 2006). As condições de uso do solo selecionadas foram: 
uma área de mata nativa (Floresta Ombrofila Densa) e quatro áreas de pastagens de Brachiaria brizantha cv. Marandu, sendo uma com 2 (P2a), uma com 9 (P9a) e duas com 18 (P18a e P18ad) anos de uso. As três primeiras pastagens (P2a, P9a e P18a) sempre foram bem manejadas e a última (P18ad) encontravase em franco processo de degradação. Vale a pena ressaltar que a pastagem com 9 anos de uso com experimento de $B$. brizantha foi, anteriormente, durante pelo menos 28 anos com pastagem de $B$. humidicola ou B. decumbens.

Nas três pastagens consideradas bem manejadas (P2a, P9a e P18a) a floresta em estádio inicial de regeneração (capoeira), foi removida por trator, amontoada e queimada. Além disso, estas áreas sempre foram conduzidas com adubação e calagem dos solos, baseadas em análises prévias e adoção de sistemas de pastejo, com carga animal adequada para cada situação. A área considerada mal manejada (P18ad) teve como vegetação antecedente, floresta em estádio inicial de regeneração, a qual foi removida pelo uso do fogo em toda a área. Esta área permaneceu com pastejo conduzido da mesma forma que as áreas bem manejadas por 12 anos, mas, no período de 1997 a 2002, passou a ser utilizada no manejo de grupos heterogêneos de animais (bovinos e equinos) da ESSUL, sem um controle rigoroso de manejo. Nesse último período, não foram realizadas adubações de manutenção e frequentemente a área era superpastejada. Pelas características apresentadas nos últimos anos de uso, como baixa produção de forragem, elevada presença de plantas invasoras e de solo descoberto, e por observação visual do pasto, esta pastagem foi considerada degradada.

As áreas foram subdivididas em três parcelas, onde foram realizadas as coletas de solo. As amostras de solo foram coletadas no período de novembro de 2002 a janeiro de 2003, nas profundidades de 0-5, 5-15 e 15-30 cm, coincidentes com o horizonte A do solo. Para a determinação da densidade do solo, foram coletadas três amostras por parcela e para a determinação do C e caracterização química e física do solo, foi coletada uma amostra composta de 20 amostras simples por parcela. O C orgânico foi determinado pelo método proposto por Yeomans \& Bremner (1988). A densidade do solo foi determinada segundo Embrapa (1997) e a abundância natural do ${ }^{13} \mathrm{C}$ por espectrômetro de massa de relações isotópicas (Finnigan DeltaPlus) acoplado a um analisador automático de $\mathrm{C}$ e N (Carlo Erba EA 1108), no Centro Nacional de Pesquisa em Agrobiologia (Embrapa). Com o objetivo de caracterizar os solos estudados, foram realizadas análises de textura, $\mathrm{pH}$ em água e complexo sortivo (Ca, Mg, K, Na, Al, H + Al, $\mathrm{S}, \mathrm{CTC}$ e V) segundo métodos propostos por Embrapa (1997). Os cálculos para a obtenção dos valores da abundância natural do ${ }^{13} \mathrm{C}\left(\delta^{13} \mathrm{C}\right)$ para as áreas de mata e pastagens seguiram o modelo descrito em Neill et al., (1996) e Koutika et al. (1997). Para a estimativa da proporção de $\mathrm{C}$ derivado da floresta, utilizou-se a seguinte fórmula:

$$
\mathrm{C}_{\mathrm{s}} \times \delta^{13} \mathrm{C}_{\mathrm{s}}=\left(\mathrm{C}_{\mathrm{f}} \times \delta^{13} \mathrm{C}_{\mathrm{f}}\right)+\left(\mathrm{C}_{\mathrm{p}} \times \delta^{13} \mathrm{C}_{\mathrm{p}}\right)
$$

em que $\mathrm{C}_{\mathrm{s}}$ é o conteúdo de $\mathrm{C}$ orgânico total (dag kg-1), $\mathrm{C}_{\mathrm{f}}$ e $\mathrm{C}_{\mathrm{p}}$ são proporções relativas do $\mathrm{C}$ da floresta e da pastagem, $\delta^{13} \mathrm{C}_{\mathrm{s}}, \delta^{13} \mathrm{C}_{\mathrm{f}}$ e $\delta^{13} \mathrm{C}_{\mathrm{p}}$ e $\delta_{\mathrm{p}}$ são os respectivos valores de $\delta^{13} \mathrm{C}$ (\%) da camada de solo total, do $\mathrm{C}$ derivado da floresta e do $\mathrm{C}$ do pasto e o valor médio do resíduo de plantas do pasto. $\mathrm{O}$ valor de $\delta^{13} \mathrm{C}$ para a vegetação de floresta foi o determinado em cada profundidade de solo amostrada nas parcelas de mata e para as gramíneas foi utilizado o valor aproximado de - $13 \%$ sugerido por Cerri \& Volkoff (1991) e Neill et al. (1996).

Para a determinação do estoque de C orgânico total $\left(\mathrm{Mg} \mathrm{ha}^{-1}\right)$, levou-se em consideração a massa do solo referente à camada de acordo com a densidade de cada uma delas. Para minimizar erros no cálculo dos estoques de $\mathrm{C}$, advindos da compactação do solo das pastagens, alguns autores os estima para a massa de solo nas condições naturais (Veldkamp, 1993; Neill et al., 1996; Tarré et al., 2001), uma vez que, quando compactadas, amostras retiradas em camadas de solo da pastagem deixam de ser diretamente comparáveis com amostras na mata na mesma profundidade.

Neste trabalho, optou-se pela correção dos estoques de C nas áreas de pasto, utilizando como referência a massa de solo encontrada em camadas equivalentes da mata, uma vez que o solo em questão apresenta variações de textura e densidade do solo ao longo do perfil, provavelmente decorrentes do uso e manejo diferenciados. Neste caso, apesar de não serem suficientes para alterar a classe dos solos, as variações encontradas entre as subcamadas retiradas dos horizontes superficiais do solo alteram os resultados obtidos, principalmente em relação ao estoque de carbono no solo.

Os resultados foram submetidos à análise de variância univariada na qual foram considerados como fontes de variação os diferentes sistemas de uso do solo (SAS, 1988). Nesse caso, consideraram-se os cinco ambientes como dispostos em delineamento inteiramente ao acaso, com três repetições. As análises de variância das variáveis de resposta foram realizadas separadamente para cada uma das profundidades de solo. Para as comparações entre as médias, foi utilizado o teste de Duncan $(\mathrm{p}<0,05)$.

\section{RESULTADOS E DISCUSSÃO}

Avaliando-se a característica física (textura) e as características químicas ( $\mathrm{pH}$ e complexo sortivo) dos solos nas áreas estudadas, podem ser observadas diferenças significativas $(\mathrm{p}<0,05)$ entre os sistemas de uso para as três camadas de solo (Quadro 1). Apesar das diferenças encontradas, estas variações não foram suficientes para alterar a classe dos solos 
nas áreas estudadas, uma vez que elas mostram apenas alterações das características entre camadas do horizonte A.

Em relação à textura do solo, as maiores diferenças foram observadas no solo do pasto bem manejado com 18 anos (P18a), que apresentou maior teor de argila e menor teor de areia nas três camadas (Quadro 1). Estas diferenças provavelmente são devidas a pequenas variações no microrrelevo, comuns em áreas dos tabuleiros costeiros. Tais variações, muitas vezes, são encontradas em áreas abaciadas com camadas superficiais um pouco mais arenosas entre si e, em áreas mais argilosas, encontradas nas bordas desses abaciados ou dos tabuleiros (caso da área P18a) pela maior suscetibilidade aos processos erosivos que tendem a remover o horizonte $\mathrm{A}$, mais arenoso e expor o horizonte $\mathrm{B}$, mais argiloso.

Vale ressaltar que, muitas vezes, esta diferenciação entre camadas não é detectada nas análises de solos para levantamento, uma vez que, nesse caso, são coletadas amostras que representam a média de todo o horizonte e não de camadas seccionadas dentro de cada horizonte, como foi realizado neste estudo.

Apesar das correções e adubações realizadas nas pastagens, as características químicas do solo não refletem diferenças acentuadas entre esses ambientes e a mata (Quadro 1). Além disso, as coletas de solo para análise realizadas neste estudo aconteceram antes da adubação de manutenção programada para áreas P2a, P9a e P18a, que geralmente ocorrem a cada três anos (em P18ad estas práticas não são realizadas há pelo menos nove anos). Assim, pode-se inferir, com base nas referências sugeridas por Alvarez V. et al. (1999), que as amostras coletadas nas pastagens, nesse período, refletem pobreza química provocada pela remoção dos nutrientes adicionados aos solos. Esta remoção está relacionada, em parte, à absorção de nutrientes pelas plantas cultivadas, e a processos de lixiviação, potencializados pelo fato de se tratar de um solo com horizonte A arenoso, em cima de um B argiloso.

Com base nas referências sugeridas por Alvarez V. et al. (1999), os teores de C, quando avaliado em base de massa (dag kg-1), encontram-se entre médio e alto nas duas primeiras camadas do solo em todas as áreas, não havendo diferença significativa entre elas (Quadro 2).

De maneira geral, os teores de $\mathrm{C}$ tenderam a ser menores nas camadas mais profundas, fato considerado normal, uma vez que os maiores aportes de matéria orgânica são observados nas camadas

Quadro 1. Características físicas e químicas das amostras de solo coletadas em áreas de mata e pastagens de B. brizantha bem manejadas com 2 (P2a), 9 (P9a) e 18 (P18a) anos de uso e mal manejada com 18 anos de uso (P18ad)

\begin{tabular}{|c|c|c|c|c|c|c|c|}
\hline Uso $^{(1)}$ & Areia & Silte & Argila & $\mathbf{p H}$ & $\mathrm{SB}^{(2)}$ & $\mathrm{CTC}^{(3)}$ & $\mathbf{V}^{(4)}$ \\
\hline & \multirow{2}{*}{\multicolumn{3}{|c|}{ dag $\mathrm{kg}^{-1}$}} & & \multicolumn{2}{|c|}{$-\mathrm{cmol}_{\mathrm{c}} \mathrm{dm}^{-3}$} & $\%$ \\
\hline & & & & \multicolumn{4}{|c|}{ Camada $0-5 \mathrm{~cm}$} \\
\hline Mata & $87 \mathrm{a}$ & $2 \mathrm{c}$ & $11 \mathrm{~b}$ & $5,3 \mathrm{~b}$ & $3,2 \mathrm{ab}$ & $13,5 \mathrm{a}$ & $23,3 \mathrm{ab}$ \\
\hline $\mathrm{P} 2 \mathrm{a}$ & $82 \mathrm{ab}$ & $3 \mathrm{bc}$ & $15 \mathrm{~b}$ & $6,0 \mathrm{a}$ & $3,1 \mathrm{ab}$ & $11,5 \mathrm{a}$ & $26,6 \mathrm{a}$ \\
\hline P9a & $80 \mathrm{~b}$ & $6 a b$ & $14 \mathrm{~b}$ & $6,0 \mathrm{a}$ & $3,7 \mathrm{a}$ & $11,8 \mathrm{a}$ & $30,9 \mathrm{a}$ \\
\hline $\mathrm{P} 18 \mathrm{a}$ & $69 \mathrm{c}$ & $6 a b$ & $25 \mathrm{a}$ & $5,5 \mathrm{~b}$ & $1,9 \mathrm{~b}$ & $12,3 \mathrm{a}$ & $15,8 \mathrm{~b}$ \\
\hline P18ad & $77 \mathrm{~b}$ & $8 \mathrm{a}$ & $15 \mathrm{~b}$ & $6,0 \mathrm{a}$ & $3,6 \mathrm{a}$ & $11,3 \mathrm{a}$ & $32,2 \mathrm{a}$ \\
\hline \multirow[t]{2}{*}{$\mathrm{CV}(\%)^{(5)}$} & 4,4 & 39,3 & 23,6 & 2,6 & 27,9 & 11,1 & 20,7 \\
\hline & \multicolumn{7}{|c|}{ Camada $5-15 \mathrm{~cm}$} \\
\hline Mata & $86 \mathrm{a}$ & $2 \mathrm{~b}$ & $12 \mathrm{c}$ & $5,1 \mathrm{~b}$ & $1,5 \mathrm{~b}$ & $10,2 \mathrm{~b}$ & $14,3 \mathrm{bc}$ \\
\hline $\mathrm{P} 2 \mathrm{a}$ & $78 \mathrm{~b}$ & $2 \mathrm{~b}$ & $20 \mathrm{~b}$ & $5,7 \mathrm{a}$ & $2,1 \mathrm{ab}$ & $10,6 \mathrm{~b}$ & $20,1 \mathrm{bc}$ \\
\hline P9a & $78 \mathrm{~b}$ & $5 \mathrm{a}$ & $17 \mathrm{bc}$ & $5,8 \mathrm{a}$ & $2,3 \mathrm{ab}$ & $10,5 \mathrm{~b}$ & $22,2 \mathrm{~b}$ \\
\hline $\mathrm{P} 18 \mathrm{a}$ & $65 \mathrm{c}$ & $6 \mathrm{a}$ & 29 a & $5,3 \mathrm{~b}$ & $1,5 \mathrm{~b}$ & $12,8 \mathrm{a}$ & $11,8 \mathrm{c}$ \\
\hline P18ad & $74 \mathrm{~b}$ & $7 \mathrm{a}$ & $19 \mathrm{bc}$ & $6,1 \mathrm{a}$ & $3,0 \mathrm{a}$ & $8,9 \mathrm{~b}$ & $33,8 \mathrm{a}$ \\
\hline \multirow[t]{2}{*}{$\mathrm{CV}(\%)$} & 5,3 & 23,1 & 20,8 & 3,7 & 26,9 & 9,3 & 24,4 \\
\hline & \multicolumn{7}{|c|}{ Camada $15-30 \mathrm{~cm}$} \\
\hline Mata & $78 \mathrm{a}$ & $2 \mathrm{~b}$ & $20 \mathrm{~b}$ & $5,0 \mathrm{~b}$ & $1,1 \mathrm{~b}$ & $10,3 \mathrm{a}$ & $10,5 \mathrm{~b}$ \\
\hline $\mathrm{P} 2 \mathrm{a}$ & $71 \mathrm{ab}$ & $2 \mathrm{~b}$ & $27 \mathrm{~b}$ & $5,5 \mathrm{ab}$ & $1,6 a b$ & $10,2 \mathrm{a}$ & $15,8 \mathrm{~b}$ \\
\hline P9a & $69 \mathrm{~b}$ & $6 \mathrm{a}$ & $25 \mathrm{~b}$ & $5,5 \mathrm{ab}$ & $1,5 \mathrm{ab}$ & $10,4 \mathrm{a}$ & $14,5 \mathrm{~b}$ \\
\hline $\mathrm{P} 18 \mathrm{a}$ & $55 \mathrm{c}$ & $8 \mathrm{a}$ & $37 \mathrm{a}$ & $5,1 \mathrm{~b}$ & $0,9 \mathrm{~b}$ & $10,8 \mathrm{a}$ & $8,7 \mathrm{~b}$ \\
\hline P18ad & $66 \mathrm{~b}$ & $8 \mathrm{a}$ & $26 \mathrm{~b}$ & $6,0 \mathrm{a}$ & $2,0 \mathrm{a}$ & $6,7 \mathrm{~b}$ & 29,9 a \\
\hline CV (\%) & 6,7 & 28,6 & 16,7 & 6,1 & 28,7 & 8,1 & 26,9 \\
\hline
\end{tabular}

${ }^{(1)}$ Sistema de uso do solo. ${ }^{(2)}$ Soma de bases. ${ }^{(3)}$ Capacidade de troca catiônica total. ${ }^{(4)}$ Saturação por bases. ${ }^{(5)}$ Coeficiente de variação. Médias seguidas pela mesma letra, na coluna, para cada uma das camadas, não diferem entre si pelo teste de Duncan $(\mathrm{p}<0,05)$ 
Quadro 2. Estoques e teores de carbono orgânico total sem (C) e com correção (Ccor) em relação à massa do solo sob mata, abundância natural de isótopos ${ }^{13} \mathrm{C}\left(\delta^{13} \mathrm{C}\right)$, percentagem de $\mathrm{C}$ orgânico derivado da floresta (Cflo) e densidade do solo (DS) em áreas de mata nativa e pastagens de $B$. brizantha bem manejadas, com 2 (P2a), 9 (P9a) e 18 (P18a) anos e mal manejada com 18 anos (P18ad)

\begin{tabular}{|c|c|c|c|c|c|}
\hline Uso & $\mathbf{C}$ & $\mathrm{C} \operatorname{cor}$ & $\delta^{13} \mathbf{C}$ & C flo & DS \\
\hline & $\mathrm{Mg} \mathrm{ha}^{-1}$ & 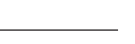 & 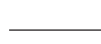 & - & $\mathrm{kg} \mathrm{dm}^{-3}$ \\
\hline \multicolumn{6}{|c|}{ Camada $0-5 \mathrm{~cm}$} \\
\hline Mata & $8,92 \mathrm{~b}(2,1 \mathrm{a})$ & $8,92 \mathrm{a}$ & $-29,08$ & $100 \mathrm{a}$ & $0,90 \mathrm{~b}$ \\
\hline $\mathrm{P} 2 \mathrm{a}$ & $17,63 \mathrm{aA}(2,6 \mathrm{a})$ & $11,08 \mathrm{aB}$ & $-20,11$ & $46 \mathrm{~b}$ & $1,39 \mathrm{a}$ \\
\hline P9a & 14,66 aA $(2,1 \mathrm{a})$ & $9,38 \mathrm{aB}$ & $-21,40$ & $55 \mathrm{~b}$ & $1,38 \mathrm{a}$ \\
\hline $\mathrm{P} 18 \mathrm{a}$ & $16,83 \mathrm{aA}(2,5 \mathrm{a})$ & $11,25 \mathrm{aB}$ & $-20,50$ & $47 \mathrm{~b}$ & $1,35 \mathrm{a}$ \\
\hline P18ad & $14,68 \mathrm{aA}(2,2 \mathrm{a})$ & $10,11 \mathrm{aB}$ & $-18,83$ & $37 \mathrm{~b}$ & $1,32 \mathrm{a}$ \\
\hline CV (\%) & 23,9 & 23,9 & -- & 27,3 & 10,3 \\
\hline \multicolumn{6}{|c|}{ Camada $5-15 \mathrm{~cm}$} \\
\hline Mata & 22,66 a $(2,0$ a) & $22,66 \mathrm{a}$ & $-26,11$ & $100 \mathrm{a}$ & $1,15 \mathrm{~b}$ \\
\hline $\mathrm{P} 2 \mathrm{a}$ & $26,26 \mathrm{aA}(1,7 \mathrm{a})$ & $19,07 \mathrm{aB}$ & $-21,59$ & $65 \mathrm{~b}$ & $1,57 \mathrm{a}$ \\
\hline $\mathrm{P9a}$ & $30,27 \mathrm{aA}(2,0 \mathrm{a})$ & $23,66 \mathrm{aB}$ & $-23,08$ & $76 \mathrm{~b}$ & $1,48 \mathrm{a}$ \\
\hline P18a & 24,74 aA $(1,7 \mathrm{a})$ & $19,69 \mathrm{aB}$ & $-22,59$ & $73 \mathrm{~b}$ & $1,46 \mathrm{a}$ \\
\hline P18ad & 22,62 aA (1,6 a) & $18,37 \mathrm{aB}$ & $-20,74$ & $59 \mathrm{~b}$ & $1,42 \mathrm{a}$ \\
\hline CV (\%) & 23,0 & 23,0 & -- & 16,1 & 5,9 \\
\hline \multicolumn{6}{|c|}{ Camada $15-30 \mathrm{~cm}$} \\
\hline Mata & $23,56 \mathrm{ab}(1,1 \mathrm{ab})$ & $23,56 \mathrm{a}$ & $-26,81$ & $100 \mathrm{a}$ & $1,40 \mathrm{c}$ \\
\hline $\mathrm{P} 2 \mathrm{a}$ & $31,82 \mathrm{aA}(1,5 \mathrm{a})$ & $30,84 \mathrm{aA}$ & $-23,10$ & $73 \mathrm{~b}$ & $1,44 \mathrm{bc}$ \\
\hline P9a & $30,70 \mathrm{abA}(1,3 \mathrm{ab})$ & 27,99 aA & $-23,96$ & $79 \mathrm{~b}$ & $1,53 \mathrm{ab}$ \\
\hline $\mathrm{P} 18 \mathrm{a}$ & $23,88 \mathrm{abA}(1,2 \mathrm{ab})$ & $24,50 \mathrm{aA}$ & $-23,88$ & $79 \mathrm{~b}$ & $1,38 \mathrm{c}$ \\
\hline P18ad & $18,88 \mathrm{bA}(0,8 \mathrm{~b})$ & $16,50 \mathrm{aA}$ & $-22,34$ & $68 \mathrm{~b}$ & $1,58 \mathrm{a}$ \\
\hline $\mathrm{CV}(\%)$ & 28,4 & 28,4 & -- & 7,5 & 3,7 \\
\hline
\end{tabular}

Médias seguidas pela mesma letra minúscula, na coluna, e maiúsculas, na linha, para cada uma das camadas, não diferem entre si pelo teste de Duncan $(\mathrm{p}<0,05)$. Valores entre parêntese na primeira coluna referem-se ao teor de carbono em base de massa $\left(\right.$ dag $\left.\mathrm{kg}^{-1}\right)$.

superficiais. Na camada de 15 a $30 \mathrm{~cm}$, esses teores estão entre médio para as pastagens P2a, P9a e P18a e baixo para mata e P18ad, havendo diferença significativa apenas entre P2a e P18ad, que

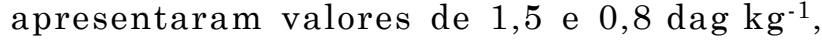
respectivamente.

Em ambientes de pastagens, diversos trabalhos (Troughton, 1951; Garwood, 1967; Silva \& Mielniczuk, 1997; Costa et al., 2000) mostram que grande parte do sistema radicular das gramíneas concentra-se nos primeiros centímetros do solo (aproximadamente $40 \mathrm{~cm}$ ), o que garante maior aporte de matéria orgânica nas camadas superficiais.

Em geral, não foram encontradas diferenças $(p<0,05)$ entre a mata e as pastagens para os teores e estoques de C orgânico total. Os trabalhos encontrados na literatura muitas vezes são contraditórios em relação às diferenças entre os teores de $\mathrm{C}$ encontrados em solos de mata nativa e pastagens. Muitos desses mostram que, pelo maior aporte de matéria orgânica proporcionado pelas raízes, os solos sob pastagens apresentam teores iguais ou superiores aos encontrados em ambientes de mata (Geraldes et al., 1995; Cerri et al., 1996; Neill et al., 1996; Fernandes et al., 1997; Marchiori Júnior \& Melo, 1999; Tarré et al., 2001), enquanto outros mostram valores mais altos nos solos de mata (Dantas, 1979; Dadalto et al., 1986; Noordwijk et al., 1997), proporcionado pelo maior aporte global de matéria orgânica.

Nesse contexto, sabe-se que, para uma mesma produção e deposição de biomassa vegetal no solo, o teor de matéria orgânica pode variar de solo para solo, tendo em vista a qualidade do material aportado e a influencia de diversos fatores sobre a microbiota do solo e, consequentemente, sobre a taxa de decomposição. Assim, deve-se considerar as características intrínsecas do substrato (qualidade), como alto teor de lignina e alta relação C/N (Coleman et al., 1989) e características externas, como baixa temperatura, deficiência em água, falta de aeração (anaerobiose), elementos tóxicos e baixos teores de nutrientes no solo (Resende et al., 2007), que inibem a ação dos microrganismos, podendo favorecer acúmulo de matéria orgânica. Obviamente, se nenhum desses fatores for limitante à ação da microbiota do solo, a decomposição de matéria orgânica é acelerada.

Avaliando a dinâmica do sistema radicular em resposta a regimes de desfolha, Corsi et al. (2001) mostram que o material orgânico advindo do sistema radicular de plantas forrageiras tropicais apresenta baixas taxas de decomposição, devido à presença de compostos resistentes à degradação (lignina, polifenóis, 
dentre outros) e uma larga relação entre carbono e nutrientes. Dessa forma, o aumento no teor de C nos solos sob pastagem pode estar mais ligado à qualidade do material orgânico a ser degradado do que à quantidade de material produzido nos diferentes ambientes, o que pode garantir maior permanência do C em um sistema em detrimento do outro.

Diante do exposto, pode-se inferir que generalizações levando em conta apenas o tipo de vegetação tornamse temerosas, uma vez que outros fatores estão diretamente ligados ao acúmulo de matéria orgânica ao solo.

Avaliando em termos de volume de solo $\left(\mathrm{Mg} \mathrm{ha}^{-1}\right)$, observou-se aumento nos estoques de $\mathrm{Cem}$ profundidade em todos os ambientes, devido à diferença na espessura das camadas, bem como à tendência de aumento da densidade, tanto com a profundidade do solo quanto com a mudança do uso de mata para pastagem (Quadro 2). Para os teores de C não corrigidos, observase que a mata apresenta o menor valor na camada de $0-5 \mathrm{~cm}$, diferindo significativamente $(\mathrm{p}<0,05)$ das pastagens. Nas camadas subsequentes não foram observadas diferenças significativas entre as áreas.

Os dados referentes à densidade do solo revelaram aumento significativo, especialmente quando a mata foi substituída por pastagens. No ambiente de mata, estes valores foram de 0,90 e $1,15 \mathrm{~kg} \mathrm{dm}^{-3}$, para as camadas de $0-5$ e $5-15 \mathrm{~cm}$, respectivamente, enquanto nas pastagens, as pressões mecânicas exercida no solo tanto no período de formação dos pastos quanto pelo pisoteio do gado, proporcionaram valores mais altos de densidade do solo nestas duas camadas, apresentando valores médios de $1,36 \mathrm{~kg} \mathrm{dm}^{-3}$ na primeira e $1,48 \mathrm{~kg} \mathrm{dm}^{-3}$ na segunda camada.

Na camada de $15-30 \mathrm{~cm}$, apesar das diferenças significativas entre as áreas, não se pode concluir que aí ainda exista influência do pastejo no aumento da densidade, uma vez que não são observadas diferenças entre os valores encontrados na mata e nos pastos P2a e P18a. Os valores mais elevados de densidade encontrados nesta camada podem ser explicados pela redução nos teores de matéria orgânica em profundidade, fato comprovado pelo maior valor de densidade e menor teor de carbono encontrado em P18ad (Quadro 2). Em trabalho de revisão a respeito do impacto animal sobre o solo, Cantarutti et al. (2001) mostram que, em geral, a compactação devido ao tráfego animal ocorre nos primeiros centímetros do solo, podendo estes efeitos ser encontrados até os $15 \mathrm{~cm}$, fato também observado neste trabalho.

Quando os teores de C foram corrigidos, considerando-se as mudanças ocorridas na densidade do solo nas áreas de pastagens, observou-se diferença significativa $(p<0,001 ; p<0,05)$ entre os valores corrigidos e não corrigidos em todos os ambientes, para as camadas de $0-5$ e $5-15 \mathrm{~cm}$. Na camada de $15-$ $30 \mathrm{~cm}$, onde não foram encontradas alterações nos valores de densidade entre os sistemas de uso, os valores não diferiram entre si. Com a correção, houve tendência dos teores de $\mathrm{C}$ encontrados nas pastagens se aproximarem daqueles encontrados na mata devido a sua redução, fazendo com que não fossem encontradas diferenças significativas para os teores de $\mathrm{C}$, entre os ambientes, nem mesmo na camada superficial.

Os dados anteriormente discutidos refletem a influência da compactação nos teores de $\mathrm{C}$ dos solos. Segundo Veldkamp (1993), quando a correção para compactação não é feita, a perda de $\mathrm{C}$ orgânico pode ser sistematicamente subestimada.

Em estudo realizado na mesma estação experimental onde foi desenvolvido este trabalho, Tarré et al. (2001), comparando os níveis de carbono no solo de áreas de pastagem de B. humidicola com 9 anos de uso e consorciada com leguminosa, com uma área de mata adjacente, também não encontraram diferenças significativas entre os sistemas avaliados, apesar da tendência dos maiores valores serem encontrados nas pastagens.

Cerri et al. (1996) avaliando a dinâmica do C após desmatamento e uso com pastagem, na região de Manaus, observaram diminuição de 20 a $30 \%$ no C total nos primeiros anos após o desmatamento e queima, havendo recuperação progressiva e, após 20 anos de uso com pastagem bem manejada, o carbono total ultrapassou o inicial em 5 a $15 \%$.

A análise da abundância isotópica do $\mathrm{C}$ no solo tem se mostrado uma ferramenta valiosa para a detecção de diferentes isótopos de carbono provenientes de sistemas de mata natural (predomínio de plantas $\mathrm{C}_{3}$ ) e de pastagens (predomínio de plantas $\mathrm{C}_{4}$ ) no mesmo solo (Neill et al., 1996; Tarré et al., 2001).

Neste trabalho, os valores médios de $\delta^{13} \mathrm{C}$ encontrados para a área de mata $(-27 \%$ ), nas três camadas do solo, estão dentro da faixa (-22 a -34 \%) correspondente às plantas $\mathrm{C}_{3}$ (Veldkamp, 1993), enquanto nas áreas de pasto os valores encontrados são maiores (média de - $22 \%$ ), indicando pequeno enriquecimento em $\mathrm{C}^{13}$ advindo do material orgânico das gramíneas, que segundo Cerri \& Volkoff (1991) apresentam valores próximos de - $13 \%$.

A partir dos cálculos da percentagem de C proveniente da floresta (Cflo), foram observadas diferenças significativas apenas entre o sistema de mata natural (100\%) e as pastagens (média de $62 \%$ até os $30 \mathrm{~cm}$ do solo). Entre os pastos, não foram observadas diferenças $(p<0,05)$ nas três camadas de solo analisadas.

Entre as pastagens, as maiores proporções de $\mathrm{C}$ derivado de plantas $\mathrm{C}_{4}$ foram observadas na camada mais superficial do solo $(0-5 \mathrm{~cm})$, fato também observado por Koutika et al., (1997) e Tarré et al. (2001). Esses autores observaram aumento gradativo nos teores de $\mathrm{C}$ derivado da floresta com a profundidade do solo em áreas de pastagem. Além disso, observaram que, abaixo dos $40 \mathrm{~cm}$ de profundidade do solo sob pastagem, apenas $7 \%$ do $\mathrm{C}$ foi derivado de 
gramíneas, sendo este fato atribuído ao reduzido aprofundamento das raízes da braquiária, induzida pelo fato de as chuvas na região extremo sul da Bahia serem relativamente bem distribuídas ao longo do ano.

De acordo com Cerri et al. (1996), as técnicas isotópicas possibilitam acompanhar o desaparecimento gradativo do $\mathrm{C}$ inicial da mata e a incorporação do $\mathrm{C}$ proveniente da pastagem. Esses autores constataram em solos da região de Manaus que, depois de 8 anos de pastagem, após o desmatamento, ainda havia $50 \%$ do $\mathrm{C}$ do solo proveniente da mata, demonstrando que grande parte da matéria orgânica da floresta permanece sob forma estável com o tipo de manejo adotado. Neste estudo, após 28 anos de uso com pastagens (P9a), ainda foi encontrada uma média de 55,76 e $79 \%$ de carbono derivado da mata, nas profundidades de 5,15 e $30 \mathrm{~cm}$, respectivamente (Quadro 2).

Diferentemente do que é encontrado na literatura, neste estudo não foi observado aumento gradativo nos teores de $\mathrm{C}$ derivado da pastagem com os anos de uso. $\mathrm{O}$ fato da pastagem com 28 anos (P9a) de uso ainda apresentar uma média de $68 \%$ de $\mathrm{C}$ derivado da floresta pode estar relacionado tanto com a maior humificação da matéria orgânica remanescente da floresta quanto com a maior proteção da mesma em relação à matéria orgânica proveniente do pasto. Neste caso, o aporte constante de material orgânico de melhor qualidade e ciclagem mais rápida do pasto bem manejado da referida área faz com que este seja preferencialmente degradado pelos microrganismos. De acordo com Neill et al. (1996), após a formação das pastagens em áreas desmatadas, o carbono derivado das gramíneas substitui rapidamente $\mathrm{o} \mathrm{C}$ derivado da floresta original como o substrato predominante para a respiração microbiana, mostrando que, nesse caso, a origem do $\mathrm{C}$ tem pouca influência na utilização microbiana da maior parte do componente lábil do C orgânico do solo.

Os valores de $\mathrm{C}$ derivado do pasto, observados logo a partir dos 2 anos (P2a), mostram-se mais elevados (média entre camadas de $40 \%$ ) que aqueles apresentados na literatura (Neill et al., 1996; Koutika et al., 1997; Tarré et al., 2001) nos primeiros anos de uso com pastagens. Este fato pode ser atribuído tanto à maior produção e ao aporte de material orgânico, de mais fácil decomposição (Monteiro et al., 2002), produzido pelo capim $B$. brizantha, em relação a outras gramíneas, quanto por características ligadas ao manejo e às características de cada região.

Em trabalho também realizado na ESSUL, Tarré et al. (2001) observaram que os solos de áreas experimentais com pastos de B. humidicola, bem manejados com 1, 7 e 10 anos de uso, formados a partir de áreas desmatadas ou com floresta, apresentaram incremento médio de $\mathrm{C}$ derivado da gramínea da ordem de 7,27 e $30 \%$, respectivamente, até a profundidade de $30 \mathrm{~cm}$. Quando os mesmos experimentos foram conduzidos a partir de pastagens mal formadas e improdutivas da mesma gramínea, com 12 anos de uso anterior, os valores registrados após 1, 7 e 10 anos de experimento foram de 12,30 e $34 \%$, respectivamente. Apesar de o segundo experimento apresentar incremento médio de $\mathrm{C}$ superior em todos os períodos avaliados, vale ressaltar que esta área já possuía um estoque de $\mathrm{C}$ derivado de gramínea anterior à instalação do experimento. Assim, esses dados sugerem que o incremento de $\mathrm{C}$ derivado da gramínea anterior ao experimento é muito menor que aquele incrementado após sua instalação, provavelmente devido ao fato do pasto anterior ser de qualidade ruim.

Em um Latossolo Amarelo da região amazônica, sob pastagens de $B$. brizantha com 7 anos de uso, Panicum maximum com 12 anos de uso e $B$. humidicola com 17 anos de uso, Koutika et al. (1997) encontraram percentagens médias de $\mathrm{C}$ derivado de gramíneas da ordem de 7,18 e $22 \%$, respectivamente, até a profundidade de $30 \mathrm{~cm}$ do solo. Nesse caso, o maior incremento de $\mathrm{C}$ está relacionado tanto com o tempo de uso com gramíneas quanto com a diferença entre as espécies utilizadas, que apresentam características distintas.

Em ambientes de pastagens com 3, 5, 20 e 81 anos de uso, cultivadas nos últimos anos com $B$. brizantha, sobre Argissolo Amarelo da região amazônica, Neill et al. (1996) encontraram contribuições relativas de C derivado de gramíneas de 13, 22, 44 e $63 \%$, respectivamente. Por outro lado, nas áreas de pastagens de $P$. maximum com 13 e 41 anos de uso, os valores encontrados foram 42 e $31 \%$, respectivamente, o que sugere que nem sempre com os anos de uso ocorrem aumentos na quantidade de $\mathrm{C}$ proveniente da pastagem.

Manfrinato et al. (2002), avaliando a origem da matéria orgânica em solos de uma planície aluvial do Quaternário (Cambissolos e Gleissolos), verificaram que, após 20 anos de uso com pastagens, $70 \%$ do C orgânico do solo ainda foi derivado da floresta original, sendo esta ocorrência explicada pelo manejo utilizado na conversão da mata para a pastagem. Segundo os autores, a retirada da floresta exportou cerca de $50 \%$ da biomassa total e o restante foi queimado ao longo dos anos de forma ineficiente, devido à alta umidade encontrada na área. Dessa forma, uma grande proporção de biomassa estável da floresta foi continuamente introduzida no solo nos primeiros $2 \mathrm{a}$ 3 anos da transformação. Além disso, os dados fornecidos pelos autores sugerem que a alta permanência do $\mathrm{C}$ proveniente da floresta ao solo, após 20 anos de uso com pastagem, pode também ser devido a reduzida taxa de decomposição da matéria orgânica, provavelmente devido à atuação ineficiente dos microrganismos no processo de decomposição, uma vez que se sabe que a alta eficiência dos microrganismos no processo de decomposição da matéria orgânica do solo depende de condições adequadas de $\mathrm{pH}$, temperatura, umidade, disponibilidade de nutrientes, dentre outras. 
Diante do exposto, pode-se inferir que apenas o tempo de uso do solo com pastagem não pode ser considerado como a única característica responsável pelo incremento de $\mathrm{C}$ derivado de gramínea aos solos. Nesse caso, o tipo de manejo e de gramínea utilizados, bem como as características ligadas ao ambiente, devem ser consideradas.

\section{CONCLUSÕES}

1. Não houve diferença significativa para os estoques de $\mathrm{C}$ do solo entre os ambientes de mata, pasto degradado e pasto produtivo, com diferentes idades de uso e nas diferentes profundidades de solo avaliadas.

2. Após 28 anos de uso com pastagem bem e mal manejada, em média $62 \%$ do C orgânico do solo ainda é derivado da floresta original até $30 \mathrm{~cm}$ de profundidade do solo.

\section{LITERATURA CITADA}

ALVAREZ V., V.H.; NOVAIS, R.F.; BARROS, N.F.; CANTARUTTI, R.B. \& LOPES, A.S. Interpretação dos resultados das análises de solo. In: RIBEIRO, AC. GUIMARÂES, P.T.G. \& ALVAREZ V., V.H. eds., Recomendações para o uso de corretivos e fertilizantes em Minas Gerais. $5^{\circ}$ aproximação. Viçosa, MG, 1999. p.2532.

APPS, M.J. Forests, the global carbon cycle and climate change. Disponível em: < http://www.fao.org/DOCREP/ARTICLE/ WFC/XII/MS14-E>. Acesso em 20 maio de 2007.

CADISCH, G.; IMHOF, H.; URQUIAGA, S.; BODDEY, R.M. \& GILLER, K.E. Carbon turnover (delta-c-13) and nitrogen mineralization potential of particulate light soil organic matter after rainforest clearing. Soil Biol. Biochem.,28:1555-1567, 1996.

CANTARUTTI, R.B.; NASCIMENTO Jr., D. \& COSTA, O.V. Impacto animal sobre o solo: compactação e reciclagem de nutrientes. In: MATTOS, W.R.S., ed.. A produção animal na visão dos brasileiros. Piracicaba, FEALQ, 2001. p.826-837.

CERRI, C.C. \& VOLKOFF, F. Nature and behaviour of organic matter in soils under natural forest, and after deforestation, burning and cultivation, near Manaus. For. Ecol. Manag., 38:247-257, 1991.

CERRI, C.C.; BERNOUX, M.; VOLKOFF, B. \& MORAES, J.L. Dinâmica do carbono nos solos da Amazônia. In: ALVAREZ V., V.H.; FONTES, L.E.F. \& FONTES, M.P.F. Os solos nos grandes domínios morfoclimáticos do Brasil e o desenvolvimento sustentável. Viçosa, MG, Sociedade Brasileira de Ciência do Solo, 1996. p.61-69.

COLEMAN, D.C.; OADES, J.M. \& UEHARA, G. Dynamics of organic matter in tropical ecossystems.Niftal Project, University Hawaí Press, 1989.
COMISSÃO EXECUTIVA DO PLANO DA LAVOURA CACAUEIRA - CEPLAC. Levantamento de solos na estação de zootecnia do Extremo Sul da Bahia. Ilhéus, CEPLAC, 1983. 31p. (Boletim Técnico, 114)

CORAZZA, E.J.; SILVA, J.E.; RESCK, D.V.S. \& GOMES, A.C. Comportamento de diferentes sistemas de manejo como fonte ou depósito de carbono em relação à vegetação de Cerrado. R. Bras. Ci. Solo, 23:425-432, 1999.

CORSI, M.; MARTHA JÚNIOR, G.B. \& PAGOTTO, D.S. Sistema radicular: Dinâmica e resposta a regimes de desfolha. In: MATTOS, W.R.S., ed. A produção animal na visão dos brasileiros. Piracicaba, FEALQ, 2001. p.838852.

COSTA, O.V.; COSTA, L.M.; FONTES, L.E.F.; ARAUJO, Q.R.; KER, J.C. \& NACIF, P.G.S. Cobertura do solo e degradação de pastagens em área de domínio de Chernossolos no sul da Bahia. R. Bras. Ci. Solo, 24:843856,2000 .

DADALTO, G.G.; COSTA, L.M. \& MOURA FILHO, W. Alterações em características químicas de solos cultivados com pastagens. R. Ceres, 3:395-403, 1986.

DANTAS, M. Pastagens da Amazônia Central: Ecologia e fauna do solo. Acta Amaz., 9:5-51, 1979.

EMPRESA BRASILEIRA DE PESQUISA AGROPECUÁRIA EMBRAPA. Centro Nacional de Pesquisa de Solos. Manual de métodos de análise do solo. Rio de Janeiro, 1997. 212p.

EMPRESA BRASILEIRA DE PESQUISA AGROPECUÁRIA EMBRAPA. Centro Nacional de Pesquisa de Solos. Sistema brasileiro de classificação de solos. 2.ed. Brasília, 2006. 306p.

FERNANDES, E.C.M.; MONTAVALLI, P.P.; CASTILLA, C. \& MUKURUMBIRA, L. Management control of soli organic matter dynamics in tropical land-use systems. Geoderma, 79:49-67, 1997.

GARWOOD, E.A. Some effects of soil water conditions and soil temperature on the roots of grasses. J. Brit. Grass. Soc., 22:176-181, 1967.

GERALDES, A.P.A.; CERRI, C.C. \& FEIGL, B.J. Biomassa microbiana de solo sob pastagens na Amazônia. R. Bras. Ci. Solo, 19:55-60, 1995.

KOUTIKA, L.S.; BARTOLI, F.; ANDREUX, F.; CERRI, C.C.; BURTIN, G.; CHONÉ Th. \& PHILIPPY, R. Organic matter dynamics and aggregation in soils under rain forest and pastures of increasing age in the eastern Amazon Basin. Geoderma, 76:87-112, 1997.

MANFRINATO, W.; PICCOLO, M.C.; CERRI, C.C.; BERNOUX, M. \& CERRI, C.E.P. Monitoring carbon stocks in soil of a forest-pasture chronosequence and determining its origin with isotope technology in Guaraqueçaba (PR). In: INTERNATIONAL SYMPOSIUM ON FOREST CARBON SEQUESTRATION AND MONITORING, Taiwan, 2002. Proceedings. Taiwan, Taiwan Forest Research Institute, 2002. p.1-10. 
MARCHIORI JÙNIOR, M. \& MELO, W.J. Carbono da biomassa microbiana, e atividade enzimática em um solo sob mata natural, pastagem e cultura do algodoeiro. R. Bras. Ci. Solo, 23:257-263. 1999.

MONTEIRO, H.C.F.; CANTARUTTI, R.B.; NACIMENTO JUNIOR, D.; REGAZZI, A.J. \& FONSECA, D.M. Dinâmica de decomposição e mineralização de nitrogênio em função da qualidade de resíduos de gramíneas e leguminosas forrageiras. R. Bras. Zootec., 31:1092-1102, 2002.

NEILL, C.; FRY, B.; MELILLO, J.; STEUDLER, P.; MORAES, F.L. \& CERRI, C.C. Forest-and pasture-derived carbon contributions to carbon stocks and microbial respiration of tropical pasture soils. Oecologia, 107:113-119, 1996.

NOORDWIJK, M.V.; CERRI, C.C.; WOOMER, P.L.; NUGROHO, K. \& BERNOUX, M. Soil carbon dynamics in the humid tropical forest zone. Geoderma, 79:187-225, 1997.

RESENDE, M.; CURI, N.; REZENDE, S.B. \& CORRÊA, G.F. Pedologia: Base para distinção de ambientes. 5.ed. Lavras, Universidade Federal de Lavras, 2007. 322p.

SAS Institute. SAS/STAT. User's guide. Release 6.03. Cary, 1988. 1028p.
SILVA, I.F. \& MIELNICZUK, J. Ação do sistema radicular de plantas na formação e estabilidade de agregados do solo. R. Bras. Ci. Solo, 21:113-117, 1997.

TARRÉ, R..; MACEDO, R..; CANTARUTTI, R.B.; REZENDE, C.P.; PEREIRA, J.M..; FERREIRA, E.; ALVES, B.J.R.; URQUIAGA, S. \& BODDEY, R.M. The effect of the presence of a forage legume on nitrogen and carbon levels in soils under Brachiaria pastures in the Atlantic forest region of the South of Bahia, Brazil. Plant Soil, 234:15-26, 2001.

TROUGHTON, A. Studies on the roots and storage organs of herbage plants. J. Brist. Grass. Soc., 6:197-206, 1951.

VELDKAMP, E. Soil organic carbon dynamics in pastures established after deforestation in the humid tropics of Costa Rica. Wageningen, Van de Landbouwuniversiteit, 1993. 117p. (Tese de Mestrado)

YEOMANS, J.C. \& BREMNER, J.M. A rapid and precise method for routine determination of organic carbon in soil. Comm. Soil Sci. Plant Anal., 19:1467-1476, 1988. 